\title{
Spatio-temporal Expression Patterns of Gelatinolytic Activity in Mouse Secondary Palates
}

\author{
Yuji Taya, Yoshihito Shimazu, Yuuichi Soeno, Kaori Sato, Hisao Yagishita, Takaaki Aoba* \\ Department of Pathology, Nippon Dental University, 1-9-20 Fujimi, Chiyoda-ku, Tokyo 102-8159, Japan
}

\begin{abstract}
Taya Y, Shimazu Y, Soeno Y, Sato K, Yagishita H and Aoba T. Spatio-temporal expression patterns of gelatinolytic activity in mouse secondary palates. Oral Med Pathol 2001; 6: 37-41, ISSN 1342-
\end{abstract} 0984.

\begin{abstract}
The present study utilizing histozymography was undertaken to localize gelatinolytic activities in the epithelium and mesenchyme of embryonic mouse secondary palate. At the stages of vertical position through elevation, the mesenchyme of the palatal shelves exhibited relatively strong gelatinolytic activities, whereas the epithelia covering the palatal shelves did not show distinctive gelatinolytic activities. The location of gelatinolytic activities in the mesenchyme changed markedly after the palatal shelves started reorientation. Modest to strong enzymatic activities were first elicited in the medial edge epithelium (MEE), when the bilateral palatal processes confronted each other prior to their contact. RT-PCR analysis also confirmed the mRNA expression of MMP-2, MMP-9, MT1-MMP, and TIMP-1 in the palatal shelves at the corresponding developmental stages. The results obtained support the view that the spatiotemporal regulation of gelatinolytic activities is a key factor to reorganization of the epithelial and mesenchymal components during embryonic palate morphogenesis.
\end{abstract}

Key words: mouse secondary palate, gelatinolytic activities, matrix metalloproteinases

Correspondence: Takaaki Aoba, Department of Pathology, The Nippon Dental University, 1-9-20 Fujimi, Chiyoda-ku, Tokyo 102-8159, Japan

Phone: +81-3-3261-8311, Fax: +81-3-3261-8969, E-mail: pathology-ndu@mqb.biglobe.ne.jp

\section{Introduction}

Congenital malformations remain an important public health issue. Cleft palates, with or without cleft lip, are among the most widely known and common craniofacial defects, which are estimated to occur in approximately one in every 500-700 live births. The long-term goals of our studies are to improve the understanding of palatogenesis at the cellular and molecular level and to elucidate intrinsic and extrinsic factors, ultimately their combinatory actions, which are responsible for the pathogenesis of cleft palate and associated craniofacial disorders (1). Matrix metalloproteinases (MMPs) represent a family of structurally and functionally related enzymes responsible for the proteolytic degradation of extracellular matrix (ECM) components. It is likely that normal morphogenesis of the secondary palate requires a specific composition and spatio-temporal distribution of ECM and MMPs (2-4). The recent work using electrophoresisbasis zymography $(5,6)$ demonstrated that the expression patterns of MMPs-2, 3, 7, 9 and 13 and their specific inhibitors, i.e., TIMPs-1 and 2 , elicit temporal variations during mouse palatal morphogenesis. In situ gelatin zymography is a technique that utilizes a gelatin-based emulsion overlay to detect and, more importantly, local- ize the gelatinase activity in underlying tissues (7-9). The present study entailed this histozymographic technique in order to gain further information about the spatio-temporal expression patterns of gelatinolytic activities during mouse palate morphogenesis. RT-PCR was also used to confirm mRNA expression of putative candidates (MMP-2 and MMP-9) and their regulators (MT1-MMP and TIMP-1) in the palatal shelves at the corresponding developmental stages, where gelatinase activities were discerned histologically.

\section{Materials and Methods \\ Animals and sampling of palatal shelves}

Tissue specimens for both histozymography and RTPCR were obtained from ICR mice. Embryonic day (E) 0 was judged to be $12 \mathrm{~h}$ prior to the morning (9:00 a.m.) on which a vaginal plug was observed. We focused our attention to the following distinct stages of palatogenesis: (I) vertical position, (II) elevation or horizontal position, (III) contact between bilateral shelves, and (IV) their fusion along the anterior-posterior axis. In order to collect palatal samples at the corresponding developmental stages, pregnant dams were sacrificed by cervical dislocation on E13.5 through E15.5; these embryonic days were 
selected on the basis of the findings previously obtained using the same animal species (10). The dissected tissue specimens were used immediately for sectioning and histozymographic examination, or were stored at $-80^{\circ} \mathrm{C}$ until used for RT-PCR.

Histozymography

The whole head of fetuses was immediately frozen and embedded in Tissue Tek O.C.T. compound (Sakura Fine Tech, Japan). Serial sections were cut at a thickness of $10 \mathrm{~m}$ on a motor driven cryostat (Kryostat, Leitz, Germany). The resulting serial sections were transferred individually in order on silane-coated glass microscope slides. Selected slides were dipped in autoradiography emulsion (EM-1, Amersham Int., UK). Once dipped, the emulsion-coated slides were allowed to gel at $4^{\circ} \mathrm{C}$ for 60 min prior to incubation in a humidified chamber at $37^{\circ} \mathrm{C}$. The emulsion contained $10 \mathrm{mM}$ calcium chloride, in addition to one of the following additives at specified concentrations: the organomercurial MMP activator 4aminophenylmercuric acetate (APMA, $1 \mathrm{mM}$, Sigma, USA) or the inhibitor 1,10-phenanthroline (4 mM, Sigma). The treated slides were developed immediately (as control) and after incubation at timed intervals up to 3 days, using D-19 developer (Eastman Kodak, Rochester) according to the manufacturer's instructions. The developed emulsion image was referred to as a histozymogram. The adjacent section placed on a separate slide was stained by haematoxylin-eosin for histologic examination.

RNA isolation and RT-PCR

At least five pairs of palatal shelves at each gestational time were collected for mRNA analysis. The bilateral palatal shelves were dissected from adjacent tissues with the aid of a fine scissors after assignment of their developmental stage under examination using a dissecting microscope. The palates assigned to stage (II) included potentially two positions, i.e., elevation and horizontal position, because it was difficult to discriminate these positions after removal of the mandible and tongue. Histologic examination of randomly selected palate specimens verified that the dissected tissue mass comprised the palatal mesenchyme and covering epithelia, which included the medial edge epithelium (MEE), as well as the neighboring putative oral and nasal epithelia. After dissection, mRNA was extracted using QuickPrep Micro mRNA Purification Kit (Amersham Pharmacia Biotech Inc, USA) according to the manufacture's direction. The RNA concentration was determined by spectrophotometric readings at 260 and $280 \mathrm{~nm}$. Amplification products were generated from $100 \mathrm{ng}$ of mRNA using one step RTPCR procedure (Ready-To-Go RT-PCR Beads, Amersham Pharmacia Biotech Inc). The primers used and the PCR conditions are shown in Table 1. PCR amplification was usually repeated 35 cycles utilizing a thermal controller (PTC-100 ${ }^{\mathrm{TM}}$, MJ Res Inc., USA). RT-PCR products were

Table. 1: Primers (sense and anti-sense) used for RT-PCR

\begin{tabular}{|c|c|c|c|}
\hline & Primers & $\begin{array}{l}\text { g Temp. } \\
\left({ }^{\circ} \mathrm{C}\right)\end{array}$ & $\begin{array}{l}\text { Product Size } \\
\text { (bp) }\end{array}$ \\
\hline MMP-2 & $\begin{array}{l}\text { 5'-GTA CTT GCC ATC CTT CTC AA } \\
\text { 5'-CCT GTT TGT GCT GAA GGA CA }\end{array}$ & 58 & 615 \\
\hline MMP-9 & $\begin{array}{l}\text { 5'-TGC GAC CAC ATC GAA CTT CG } \\
\text { 5'-CCA GAG AAG AAG AAA ACC CTC TTG G }\end{array}$ & 60 & 700 \\
\hline MT1-MMP & $\begin{array}{l}\text { 5'-ACC TAC GTA CCC ACA CAC AG } \\
\text { 5'-AAA TTC TCC GTG TCC ATC CA }\end{array}$ & 55 & 661 \\
\hline TIMP1 & $\begin{array}{l}\text { 5'-TGC ACC TGT GTC CCA CCC CAC CCA CAG ACG } \\
\text { 5'-GGC TAT CTG GGA CCG CAG GGA CTG CCA GGT }\end{array}$ & 55 & 552 \\
\hline ß-actin & $\begin{array}{l}\text { 5'-CTC AAT GTC ACG CAC GAT TTC } \\
5^{\prime}-\text { GTG GGG CGC CCC AGG CAC CA }\end{array}$ & 58 & 540 \\
\hline
\end{tabular}

separated on $2 \%$ agarose gels containing ethidium bromide and visualized under UV.

\section{Image analysis}

Photography was made with a CCD digital camera using Image-Pro Plus 1.3 software. A computer-generated image of the histozymogram was superimposed onto the image of HE-stained adjacent section. Digital images of histozymograms and HE-stained microphotograms obtained from the corresponding areas were superimposed with the aid of Adobe Photoshop ${ }^{\circledR}$ 4.0.

\section{Results}

Figs. A and B show a pair of HE-stained microgram and histozymogram, which were prepared from the continuous frontal sections of a mouse embryo at E13.5. The bilateral palatal shelves were at the vertical position. Sections incubated with the emulsion containing only $\mathrm{CaCl}_{2}$ produced marginal digestion of the emulsion overlay after incubation overnight (data not shown). The addition of the activator APMA to emulsion sol gave rise to a rapid and continuous pattern of emulsion digestion. Clear areas of the emulsion overlay were interpreted as representing areas of gelatinase activity, while dark areas as lacking gelatinase activity (Fig. B). The selective digestion above the Meckel's cartilage as well as the mandible became prominent during the first $6 \mathrm{~h}$ of incuba- 

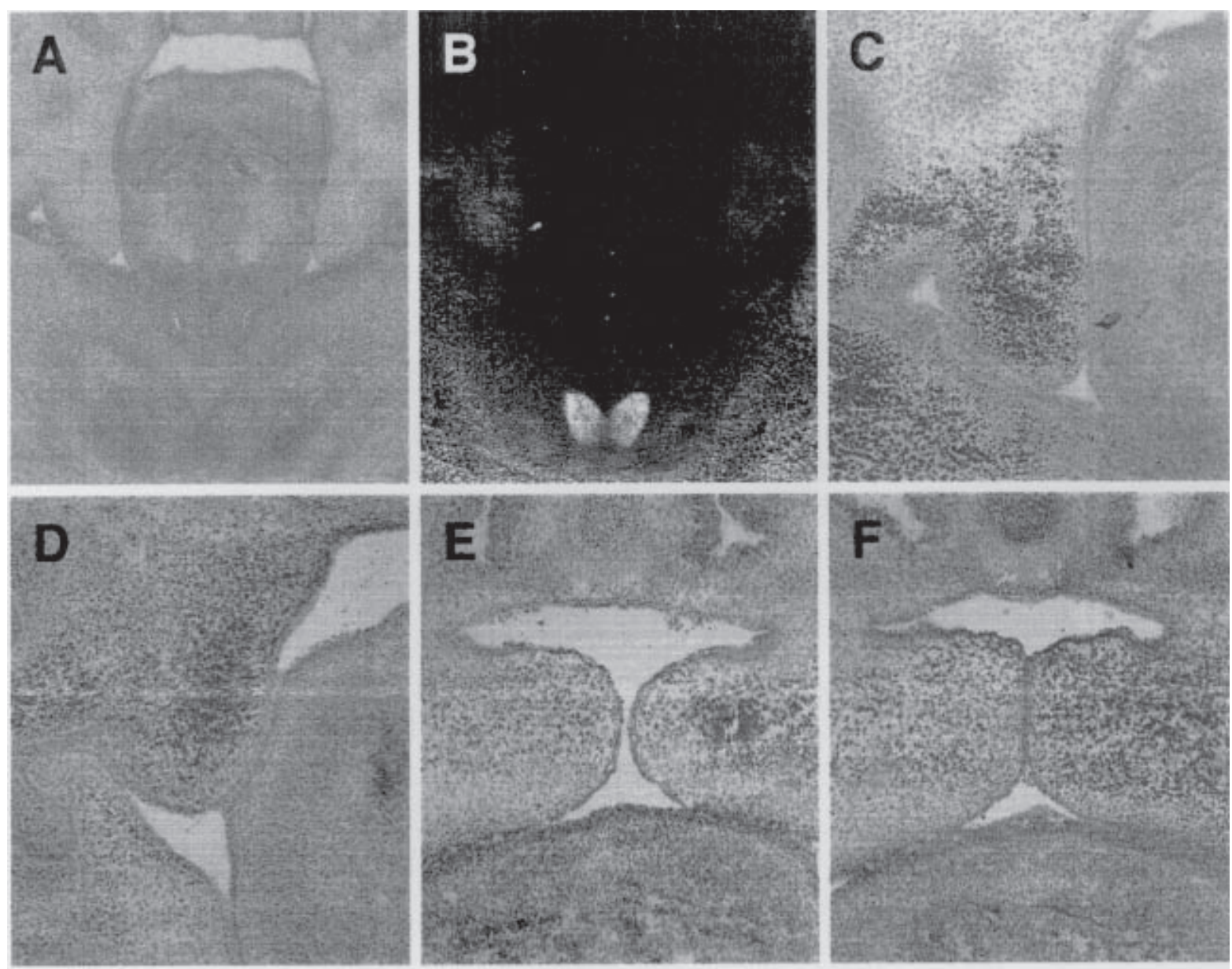

G

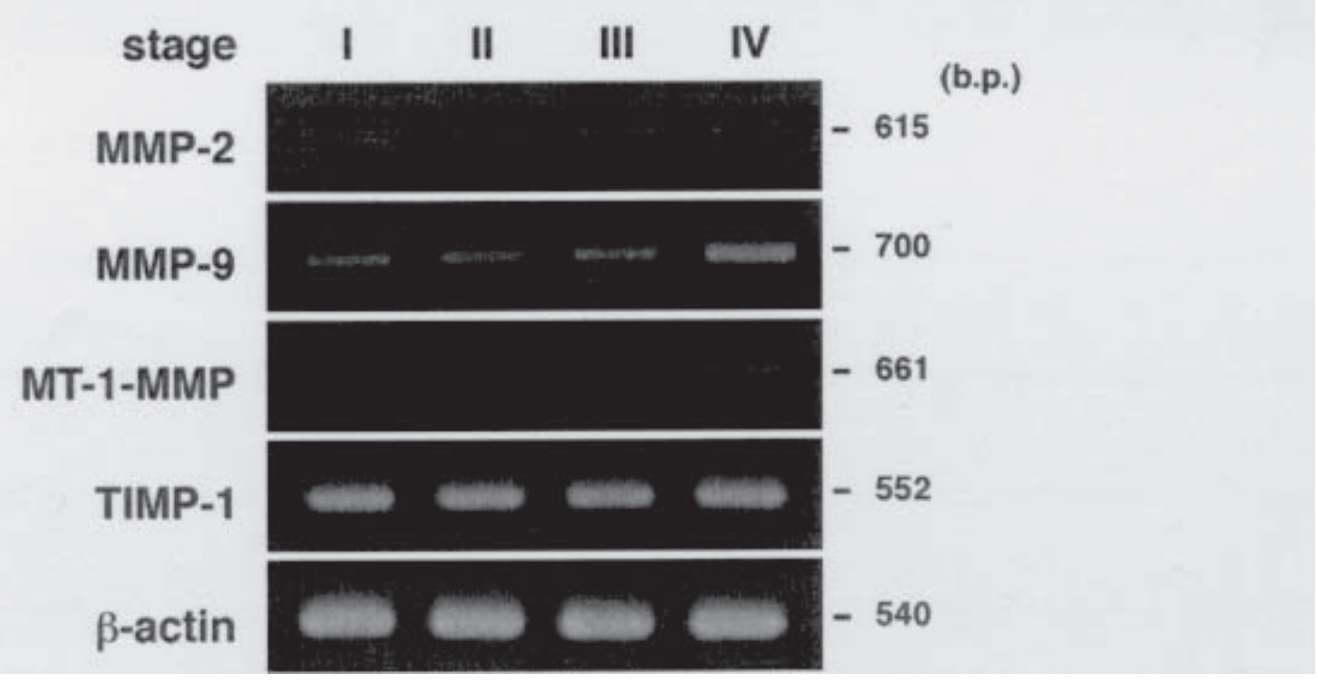

Fig. A: Photomicrogram of a frontal section of E13.5 mouse fetus, showing that the bilateral palatal shelves were at the vertical position (stage I).

Fig. B: Original histozymogram prepared from the adjacent section after incubation for $6 \mathrm{~h}$.

Fig. C: A computer-constructed image by superimposing (A) and (B); spots of gelatin digestion on histozymography are represented red in color.

Fig. D through F: The superimposed images obtained from the palatal shelves at the stages of II through IV, respectively: stage II, reorientation to horizontal position; stage III, close to contact between the bilateral MEE cells; stage IV, formation of the epithelial seam after the epithelial fusion.

Fig.G: Results of RT-PCR analysis regarding the expression patterns of MMP-2, MMP-9, MT1-MMP, and TIMP-1 in the palatal shelves at the corresponding developmental stages. PCR products were run on $2 \%$ agarose gel with ethidium bromide. The bp sizes of the individual products are indicated. Note that TIMP-1 mRNA level was the highest in intensity, while MT1-MMP was the lowest in comparison with the housekeeping gene $\beta$-actin mRNA levels. The stage-dependent variations for their expression levels were not certain, except for increasing MT1-MMP at the fusion stages. 
tion, while the tongue and the tooth organs at the cap or bell stages showed negligible or very low gelatinolysis, although weak reactions were discernible after prolonged exposure. In comparison with the gelatinolytic reaction in these surrounding tissues, the palatal shelves were found to display moderate activities. In preparating histozymograms, we always paid attention to yield relatively constant lightening for the region of Meckel's cartilage, which was used as an internal reference for assessment of the relative gelatinase activity from areas to areas within the same histozymogram. In control preparations with the addition of the inhibitor, it was also verified that there occurred no appreciable digestion in Meckel's cartilage and palatal shelves even after longer incubation (1 to 3 days).

Superimposition of the histozymogram over the corresponding microgram helped us to localize gelatinase activity generated by various cell populations. For the sake of convenience, the location of enzymatically hydrolyzed gel was represented by generation of pseudo-color in red. At the stage of vertical position (Fig. C), the mesenchyme of the palatal shelves exhibited relatively strong gelatinase activities in a rather diffuse manner, whereas both epithelia covering the palatal shelf and adjoining tongue did not show any distinctive gelatinolytic reactions. Reorientation of the palatal shelves produced slight deformation in shape due to unbalanced pressure provided by the shelf-movement along the side surfaces of tongue. The areas of gelatin digestion became expanded mainly toward the naso-palatal region and, most conspicuously, the core region of strong gelatinase activity was shifted to the underlying mesenchyme beneath the palatal epithelium in contact with the tongue epithelium (Fig. D). Notably, red spots were overlapped on some of the palatal epithelial cells at the free edge, whereas no gelatin digestion was detected for the adjoining palatal and lingual epithelia. Once the bilateral palatal shelves reached the horizontal position, thereafter approaching each other, the MEE cells started to express gelatinase activity, although no gelatin digestion was discernible for the oral-side epithelium as well as tongue epithelium (Fig. E). Similar expression of gelatinase activity was detected along the epithelial seam generated after fusion of the bilateral MEE cells (Fig. F). The overall enzymatic activities obtained in the palatal region including the MEE and mesenchymal components were apparently stronger than those in the adjacent nasal- and oral-regions.

The results of RT-PCR analysis proved mRNA expression of some putative MMPs and inhibitors in the palatal shelves (Fig. G). MMP-2 and MMP-9 messages were shown to be constitutively expressed through the developmental stages of I through IV, while a trend of increasing intensities for MT1-MMP close to the fusion between bilateral shelves (stages III and IV). Notably, the apparent message of TIMP-1 was obtained for all tissue samples at the various developmental stages.

\section{Discussion}

The present studies utilizing histozymography showed that gelatinolytic activities are implicated in the development and reorganization of mouse palatal. According to the current experimental procedure of gelatin-digestion, the precise comparison of gelatinase activity at corresponding sites between fetus specimens, or even at various sites within a single histological section, is not easy because multiple experimental factors affected the quality and black/white contrasts of histozymograms. Despite of this limitation, however, the use of histozymography gave a new insight into the sites and timings of gelatinolytic activities operating during mouse palatogenesis. Regarding the site-directed expression of gelatinolysis observed in the mandible and surrounding tissues, the present results obtained are generally consistent with the findings reported by Chin and Werb (4), showing little or no expression of MMPs and their tissue inhibitors (TIMPs) in the epithelium of tooth buds, tongue or oral cavity, but strong expression of MMP2 mRNA transcripts in the perichondrium of Meckel's cartilage and mesenchymal areas of E13-15 mandibles and MMP9 and TIMPs-1, 2 mRNAs in the ossifying areas of the mandibles.

A unique event of palatogenesis is reorientation or elevation of the bilateral palatal shelves from their vertical to horizontal position, but there remain controversies as to the mechanism or driving force of the reorientation event. It was documented that ECM components within the palatal shelves cause rapid changes in their distribution during the reorientation process $(3,11)$, which is supposed to be accomplished within several minutes to hours in murine $(10,12)$. From the present observation by histozymography, it was found that gelatinase activities in the palatal mesenchyme are distributed rather ubiquitously at the vertical position, but, concomitant with reorientation of the palatal shelves, their distribution starts to change rapidly, localizing in regions close to the concaved side facing to the tongue. This implies that the reorganization of extracellular matrix components is most likely not the result of de novo synthesis but is mediated in part by the localized activation of gelatinases, which are most likely maintained within the ECM. One possible explanation as to the rapid transition of gelatinase activity is that any alterations in cell shape and associated physical stresses may modulate processings and activation of pro-MMPs. In this connection, it is noteworthy that membrane-associated MMPs, such as MT1-MMP that was identified by RT-PCR, are known to play an important role in the activation of progelatinases, as well as regulate the degradation of a broad range of ECM molecules (13).

Another remarkable finding is the timings and lo- 
cation of gelatinolytic expression in the MEE covering the approaching palatal shelves. The palatal epithelia at the early stages I and II remained mostly negative for enzymatic activities. It is also notable that no specific gelatinolysis occurred at the sites of heterotypic epithelial contacts, such as between the lining palatal epithelial cells and the adjacent tongue epithelia, whereas weak gelatinolytic reactions were discernible at the free rims of the corresponding epithelia. In sharp contrast, when the bilateral palatal shelves confronted each other prior to their contact, the MEE cells as well as the putative nasal epithelial cells started to exhibit strong enzymatic activities. The gelatinolytic expression by the MEE cells most likely contributes to the sequential steps of palatal fusion, namely, reorganization of basement membrane, and dynamic movement and migration of epithelial cells leading to formation and subsequent disintegration of the epithelial seam. In relation to this issue, a recent report (5) substantiated the correlation between the temporospatial distribution of components of the palatal epithelial basement membrane (e.g., laminin, collagen IV and collagen III) and the expression patterns of MMP-2 and MMP-9 during shelf remodeling. Indeed, our analysis by RT-PCR also confirmed the expression of both MMPs-2 and 9 , both of which are known to degrade basement membrane components (4). At present, regulation of protease secretion by epithelial cells is not yet well understood, but it is believed that multifaceted interactions of epithelial cells with ECM, mesenchymal cells, and growth factors are implicated in this regulation $(14,15)$. Of particular interest, Miettinen et al. (16) demonstrated that MMP activity is controlled by EGF-receptor signaling pathway and that defective palate closure, due to impaired EGF-receptor signaling, results at least in part from decreased MMP activity.

In conclusion, the present study provided evidence, on the basis of histozymography, that gelatinases are localized within the palatal shelves in a developmentalstage dependent manner. More specifically, the MEE cells start to express the strong gelatinase activity prior to contact between the approaching bilateral shelves. The current experimental design did not produce solid information as to the molecular mechanism of the observed gelatinolytic activities, but RT-PCR analysis of the corresponding palatal specimens confirmed the expression of MMPs and regulators. These results are consistent with the paradigm that the interrelationships of MMPs and their activators and inhibitors provide an interwined set of action-reaction feedback loops that determine the character and three-dimensional structure of ECM components in palate development.

\section{Acknowledgment}

This work was supported in part by a Grant-in-Aid for Scientific Research (\# 12771097,\#13307052) from the
Ministry of Education, Science and Culture of Japan.

\section{References}

1. Taya Y, Soeno Y and Shimadzu Y, et al. Palatogenesis and cleft palate formation in mice: related genes and molecules. Jpn J Oral Biol 1999; 41: 531-39.

2. Ferguson MWJ. Palate development. Development 1988; 103: 41-60.

3. Morris-Wiman J and Brinkley L. An extracellular matrix infrastructure provides support for murine secondary palatal shelf remodelling. Anat Rec 1992; 234: 575-86.

4. Chin JR and Werb Z. Matrix metalloproteinases regulate morphogenesis, migration and remodeling of epithelium, tongue, skeletal muscle and cartilage in the mandibular arch. Development 1997; 124: 1519-30.

5. Morris-Wiman J, Du Y and Brinkley L. Occurrence and temporal variation in matrix metalloproteinases and their inhibitors during murine secondary palatal morphogenesis. $J$ Craniofac Genet Dev Biol 1999; 19: 201-12.

6. Morris-Wiman J, Burch H and Basco E. Temporospatial distribution of matrix metalloproteinase and tissue inhibitors of matrix metalloproteinases during murine secondary palate morphogenesis. Anat Embryol 2000; 202: 129-41.

7. Mungall BA, Pollitt CC and Collins R. Localisation of gelatinase activity in epidermal hoof lamellae by in situ zymography. Histochem Cell Biol 1998; 110: 535-40.

8. Abiko Y, Kutsuzawa M and Kowashi Y, et al. In situ detection of gelatinolytic activity in developing craniofacial tissues. Anat Embryol 1999; 200: 283-7.

9. Lee ER, Murphy G and El-Alfy M, et al. Active gelatinase B is identified by histozymography in the cartilage resorption sites of developing long bones. Dev Dyn 1999; 215: 190-205.

10. Fujiwara M, Taya Y and Aoba T. Time-sequential cellular dynamics and phenotypic changes of medial edge epithelial cells during mouse secondary palatogenesis. Jpn J Oral Biol 2000; 42: 268-82.

11. Morris-Wiman J and Brinkley L. Rapid changes in the extracellular matrix accompany in vitro palatal shelf remodelling. Anat Embryol 1993; 188: 75-85.

12. Ferguson MWJ. Palatal shelf elevation in the Wister rat fetus. J Anat 1978; 125: 555-77.

13. d'Ortho MP, Stanton H and Butler M. MT-1MMP on the cell surface causes focal degradation of gelatin films. FEBS Lett 1998; 421: 159-64.

14. Woessner JR. Matrix metalloproteinases and their inhibitors in connective tissue remodeling. FASEB J 1991; 5: 214554

15. Ries $\mathrm{C}$ and Petrides PE. Cytokine regulation of matrix metalloproteinase activity and its regulatory dysfunction in disease. Biol Chem 1995; 376: 345-55.

16. Miettinen PJ, Chin JR and Shum L, et al. Epidermal growth factor receptor function is necessary for normal craniofacial development and palate closure. Nat Genet 1999; 22: 69-73.

(Accepted for publication May 14, 2001) 